LA-UR -82-102

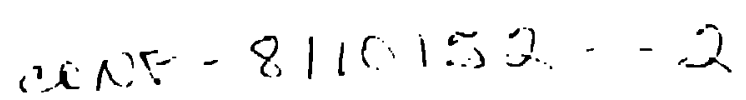

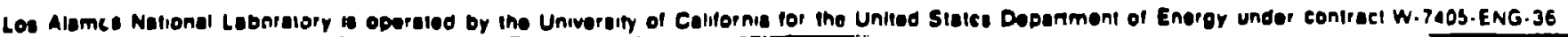

LA-UR-- $\$ 2-102$

DE\{ 2010413

TITLE: Pion Production by Target Emission

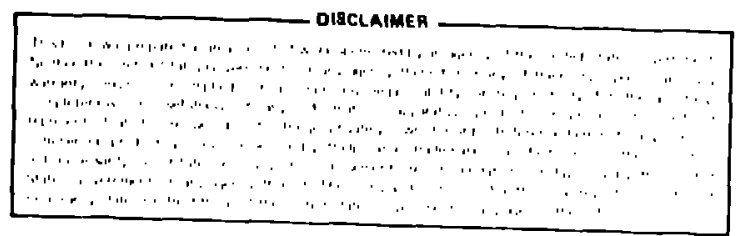

ALIMOR(S) W, R.

SUBMITTED to Presented at Workshop on Pion Production, Bloomington, Indiana $10 / 22-24 ! 81$ 


\section{PION PRODUCTION BY TARGET EMISSION}

by

W. R. Gibbs

Los Alamos National Laboratory

Los Alamos, New Mcxico 87545

\section{ABSTRACT}

The coherent production of pions in a proton nucleus collision is calculated. The energy region considered is below the nucleonnucleon threshold so that the entire nucleus must take part in the process. The amplitude for this reaction is broken into two parts, the first part being characterized by initial emission of the pion from the projectile and the second part by initial emission from the target nucleus. It is the second part which is emphasised in this talk. The target nuclei ${ }^{12} \mathrm{C}$ and ${ }^{209} \mathrm{Bi}$ are considered. Total production cross sections as a function of energy and sume angular distributions are calculated.

\section{INTRODUCTION}

Let me begin by trying to put my talk in perspective. To do this I will consider only the one pion rescattering graph. It is known 1 that a reasonable representation of the $p+p \rightarrow \pi+J$ reaction can be achieved with this type of calculation. This is not to deny the possible importance of $p$ - exchange ${ }^{2}$, interacting $\Delta^{\prime} s{ }^{3}$, relativistic effects 4 etc. but to try to take the simplest graph for use in the nuclear calculation. Taking atatic pion approximation imnediately we may writc

$$
M=-2 \sum_{\bar{q}, i, j} \frac{\left(\bar{q} \cdot\left|H_{s}^{i}\right| \bar{q}\right)\left(\bar{q}\left|H_{L}^{j}\right| 0\right)}{w(q)} \text {. }
$$

Here $i$ and $f$ label the nucleons involved in the reaction, $\bar{q}$ is the final pion momentum and $\overline{7}$ the intermediate pion momentum. $H^{j}$ is the production vertex operator and $H^{1}$ is the corresponding rescaflering; vertex opedator. The nuclear states have becn suppressed but are implicit $\left[E_{g} .10\right)$ means proton incident on ${ }^{12} \mathrm{C}$ and no pions etc.]. The rescattering operator can be related to the pion nucleon t-natrix and the production vertex is related to $\gamma_{5}$.

To obtain the two nurleon model 5 onte calculates firat a pion production "potential" by doing the qum (integra)): 


$$
v_{i j}=v\left(\bar{r}_{i}-\bar{r}_{j}\right) \sim \int d \bar{q} \frac{t\left(\bar{q}, \bar{q}^{\prime}\right) \bar{\sigma} \cdot \bar{q} e^{i \bar{q} \cdot\left(\bar{r}_{i}-\bar{r}_{j}\right)}}{w^{2}(q)} .
$$

One can then calculate the expectation value in the nucleus of this potential

$$
M \sim\left(\bar{q}^{\prime} \mid \Sigma v_{i j}(0)\right.
$$

This is a perfectly reasonable way to proceed but I shall consider another organizaion of the work. We may note that in the $i, j$ sum either $i$ or $j$ must be the projectile. If $j$ is the projectile then $i$ just labels the target nucleons. We may recognize

$$
\sum_{i=1}^{A}\left(\bar{q}\left|H_{s}^{i}\right| \bar{q}\right)
$$

as the single scattering approximation to the pion nucleus t-matrix. If we improve the calculation by using the complete multiple scattering t-matrix and add in the plane wave part we recognize this result as

$$
M \sim\left(\phi_{\pi}(\ddot{q})\left|H_{p}^{p}\right| 0\right)
$$

which is the usual one nucleon DWIA matrix element.

What about the other part? Let us consider the case where $i$ labels the projectile. In this case the shell structure is more cumplicared. I will use the ${ }^{12} \mathrm{C}$ target as a specific example. Tae final state will be some state in ${ }^{13} \mathrm{C}$ and we can write it as an expansion of the final captured nucleon multiplied by core states of ${ }^{12} \mathrm{C}$

$$
\left.I^{13} C\right\rangle=\Sigma C_{\ell j}^{J} E^{T}|J T E\rangle|\ell j\rangle .
$$

Tbis part of M will now be

$$
\sum_{1 E} \int_{12} d \bar{q} \frac{\left(\bar{q} \ell \ell j\left|H_{s}^{p}\right| \bar{q} \psi_{p}^{\prime}\right)\left(\bar{q} J T E\left|H_{D}^{i}\right| 0\right)}{w(q)} c_{\ell j}^{J T E} .
$$

Since $H^{i} \sim \tilde{\sigma}_{j} \cdot \dot{q}^{-i} r^{i}$ the core states must be unnatural parity $\left(0^{-}, 1^{+}, \sum, \ldots\right)$ and heve isospin one. Note that the matrix element on the left iefers only to the projectile and $f$ inal nucleon and the watrix element on the right contains only tar el coordinates. In order to make further progress let us expand the ${ }^{12} \mathrm{C}$ core states in particle-hole promotions from the ${ }^{12} \mathrm{C}$ ground state $j$.e.

$$
\left.|J T E\rangle=\left.\Sigma \quad B_{\ell_{h} J_{n} \ell_{p} J_{p}}^{J}|| \ell_{n}\right|_{h}>\mid \ell_{p}, v_{p}>\right\} \mathrm{JT} .
$$


Now the production matrix element can be written as

$$
\begin{aligned}
& \left(\bar{q} J \text { T E } \mid H_{p}^{j}(0) \sim \sum B_{\ell_{h} j_{h} \ell_{p} j_{p}} \frac{1}{\sqrt{w(q)}} Y_{j}(\hat{q}) Q_{p h}^{J}(q) a_{J L}\right. \\
& Q_{p h}^{J L}(q)=\operatorname{af}\left(q^{2}\right)\left(\frac{g}{m_{\pi}}\right) \hat{c}_{p h}^{J} \int_{0}^{\infty} d r r^{2} j_{L}(q r) R_{p}(r) R_{h}(r)
\end{aligned}
$$

where $\alpha=2$ for nucleons, $\alpha=8 / 3$ for $\Delta$-isobars. This last factor of $\alpha$ takes account of the fact that we could also excite $2 \Delta$-hole component. The reason for writing the function in this way is to make connection with the work of Toki and Weise, 5 and the Lyou group ${ }^{7}$ as well. Toki and heisf show how the $Q$ function nay be corrected by means of RPA to include the effects of muitiple particle-bole and delta-hole states. If we represent the simplest form of $Q$ as defined above as

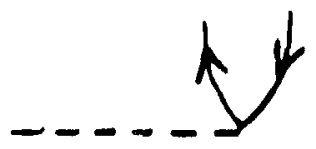

then, taking into account the possiblity of exciting virtual particje-hole states ty either pion exchange or heavy meson exchange, the $Q$ gets replaced by $Q$ where $\widetilde{Q}$ can be represented by a sum of diagrams.<smiles>CC(C)C(C)C(C)C</smiles>

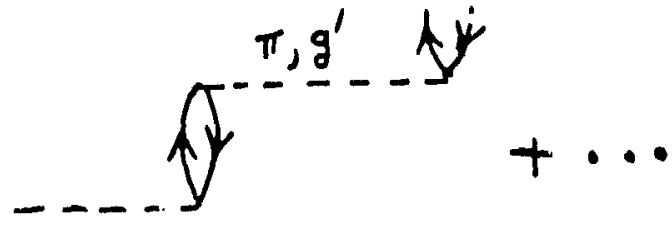

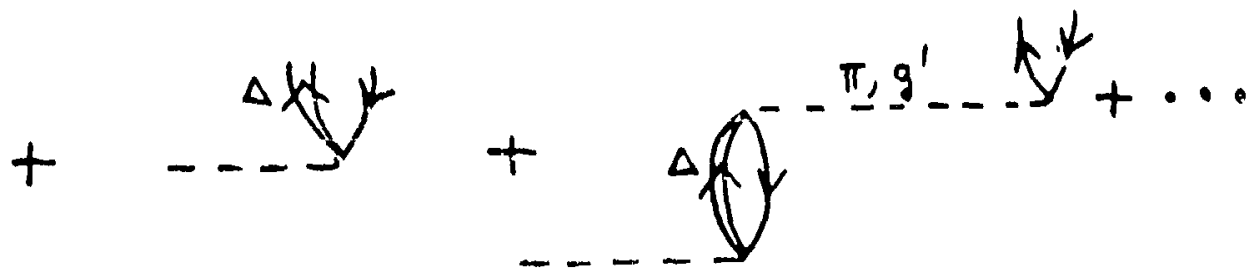

Note that, depending un one point of view, this correction can be regarded as correction to the basic particle-hole wave function or modificaicion to the pjon propogalor. The main effect in any case is to give more support to the high momentum cumponints. The rest of the nucleons in the nucleus "back up" the simple particle-hole wave function so that the plon-nucleus vertex is given enhancement from the coherent many-hody effects. This actually provides a more-than-two nucleon theory of pion production. 
Leaving of $f$ the spin and isospin indicies one can firite a typical element in the sum for $M$ as the deceptively simple expression

$$
\int \mathrm{d} \bar{r} \phi_{\pi}^{(-)^{*}(\bar{r})} \Psi_{\mathrm{f}}(\bar{r}) \text { f }\left(\bar{q}, \bar{q} \cdot \bar{p}, \bar{p}^{\prime}\right) A(\bar{r}) \Psi_{j}(\bar{r})
$$

where

$$
\begin{aligned}
& \Psi_{i}(\bar{r}) \text { is the initial proton wave function, } \\
& A(\bar{r}) \text { is the function } Q_{p h}^{J L}(q) \text { in r-space, } \\
& \dot{f}\left(\bar{q}, \bar{q}^{\prime}, \bar{p}, \bar{p}^{-}\right) \text {is a function derived from the pion-nucleon } \\
& \text { t-matrix as an operator in all four momenta, } \\
& \Psi_{f}(\bar{r}) \text { is the final bound nucleon wave function, } \\
& \phi_{\pi}^{(-)}(\bar{r}) \text { is the final pion wave furction. }
\end{aligned}
$$

This has the form of a DhIA matrix elsment for kuock out of - pion followed by a cafture of the incident nucleon. The reason that the computation of this integral is not simple is the presence of the operators $\bar{q}, \bar{q}^{\prime}, \bar{p}, \bar{p}^{\prime}$ in $f$. Here $\bar{q} \dot{q}$ the $q$ radient operator on $A(\bar{r}), q$ is the gradient cperator or $\phi(\bar{r}), \bar{r}), \bar{p}$ is the gradient operator on $\psi_{i}(\bar{r})$ and $\bar{p}^{\prime}$ is the gradient operatur on $\psi_{f}(\bar{r})$. It is only the assumption of a farly simply form for $f$ that makes computation possible. The expression used for $f$ is

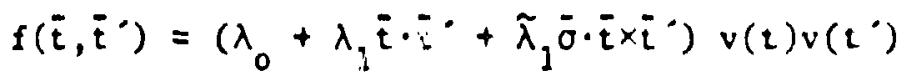

where

$$
\bar{t} \equiv \bar{q}-\frac{\mu}{m} \bar{p} \text { and } \bar{t} \equiv \dot{q}-\frac{\mu}{m} \dot{p}^{\cdot} \text {. }
$$

A schematic representation of this integral 28 given in $f i g .1$. The initial proton interaction is a sandard (ncn-rejativisitic) proton nucleus optical potential. B The pion "wdve functicn" wiss calculated with the methods of Tokj and Weike. The needed shell model coefficients were taken from the Lus Alamos version of Glargow code 10 as implemented by Kaxton and Dubach. 11 The final nucleon bound tate wave functions were solutions in a Saxon-fioods potential. The hell model coefficients were obtained as above. The final pion wave function was taken from the coordinace space nonlocal optical model code developed in T-5 al Los Alatms. 12 


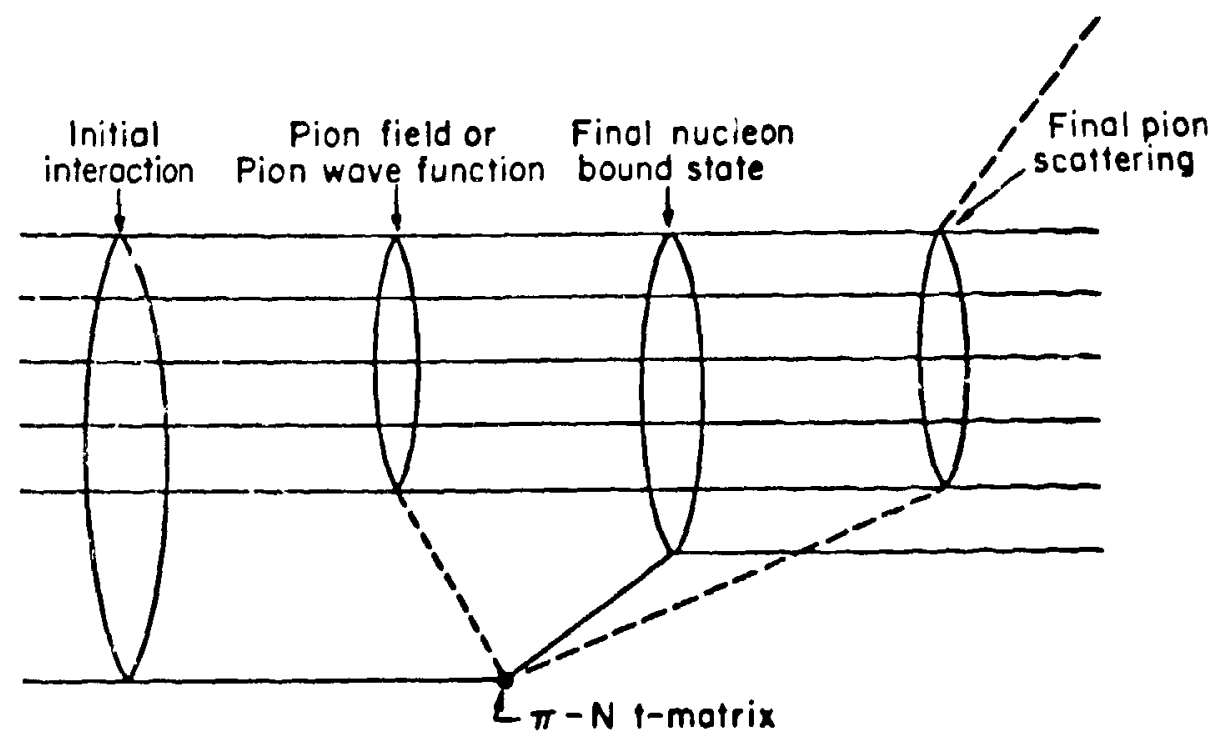

Figure 1. A schematic representation of expression 11.

I am going to consider oniy the target enission in most of what follows. The reasons for this are several. The first motivation is to separate the problem into manageable pieces and to study them independently. Since $\pi$ production may well be dominated by target emission there is a chance to look at it (almost) alone. Another reason is the hope tbat the sensitivity to the final optical potential will be less than in the projectile emission since more vertices are present in the target emission. Another sensitivity in the projectile emission is due to the treatment of true pion absorption with an incident off-shell pion. Presumably the possiblity of reabsorption is much greater since l.e momentum of the pion is nuch nearer the pole for trie absorption in the optical putentiai. 13 This would affect the projectile emission more than the target enission brcause in the latter the pion is more likely to be on-shell when it leaves the $\pi$-nucleon t-matrix.

\section{ENERGY DEPENDENCE FOR ${ }^{12} \mathrm{C}\left(\mathrm{p}, \pi^{+}\right)^{13} \mathrm{C}$}

There are many possible calculations that could be done with this technique. I will try to focus primarily on a single simple point, nanely the energy dependence of the total pion production cross section. This data is now avalable nn large number of nuciei but for the first comparison with experiment I shall reatrict myself to several states of $13 \mathrm{C}$. It was noted by the Indiana group 11 that there seemed to be two types of encrgy dependence of the total cross section; nne in which there was an flitiol rapic rise with "bend" to give a much more slowly rising function and second type which simply increased from zero with no abrupt chance in slope. The first type of behavior seemed to be asociated with single particle states and the gecond type with 2 plh states. An exception to this rule is the $9 / 2^{7}$ state at 9.5 MeV. 
Since simple projectile emission populates single particle states only, while target emission populates single particle and 2 plh states we might conjecture that the separation of reaction mechanisms might be made in this way.

The first step in this investigation was the calculation of a total cross section with projectile emission, figure 2 shows te results of a calculation using the Keating-hills-Tsangarede: 25 code.

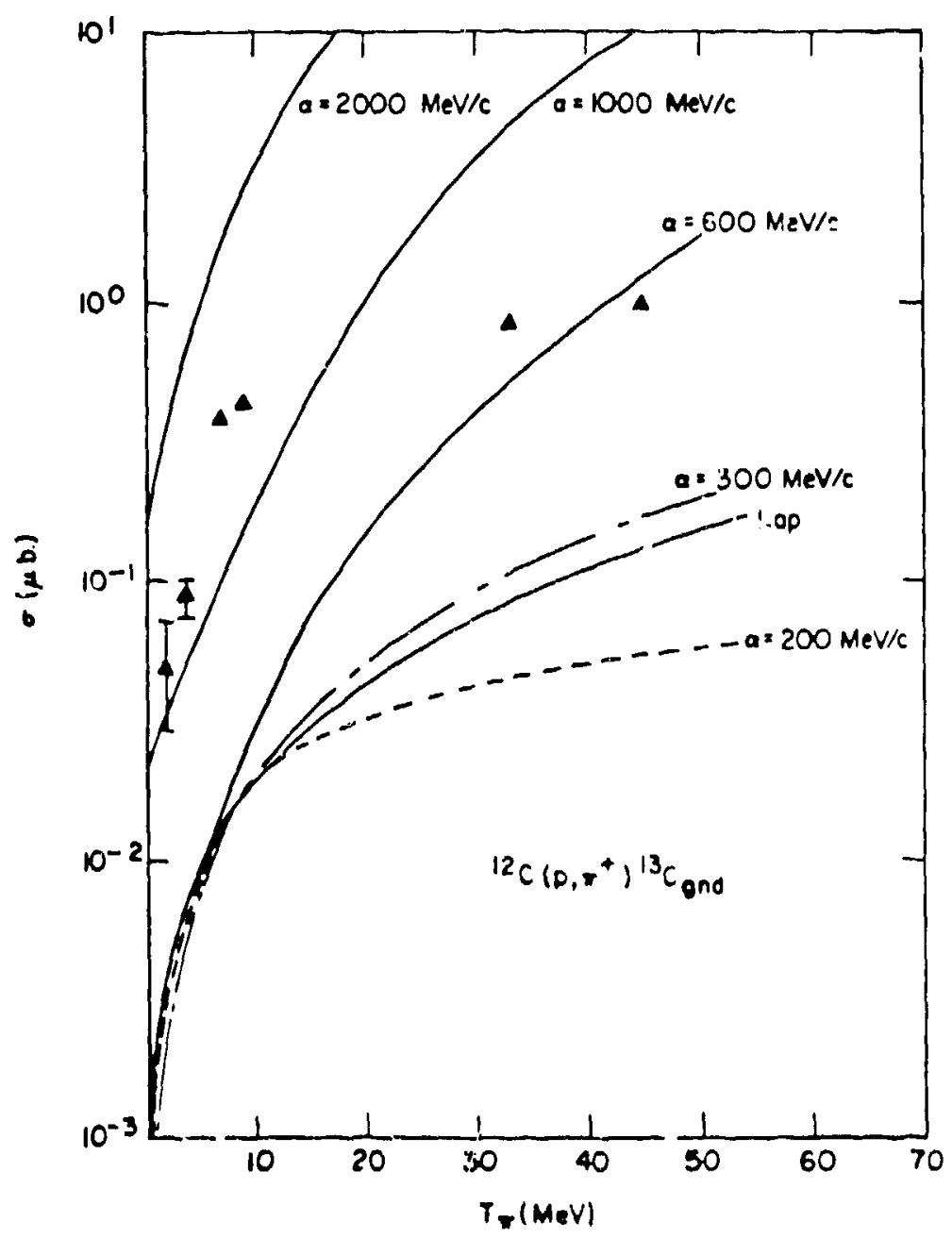

Fig. 2. One-nucleon-model calculations showing the dependence on the non-locality of the optical potential Note that this non-locality is a total effective value inlcuding e.g. the wotion of the $\Delta$.

The curve labeled "Lap" was calculated usirg the damped Laplacian furm. The other curves were calculated using the T-5 non-local code with the off-shell range denoted as a. One might expect a similar variation with other uptical model parancters, buch as $p^{2}$ terms. 
We may see that (within limits) the very low energy part is little affected by the optical potential and the variation is largely controlled by phase space and Coulomb penetrability. We note that a change in slope can be obtained at about the right exergy. Thus the hoped-for separation hypotheses is still alive although it is somewhat disturbing that the calculated cross section showing the desired shape is so far below the data. The uncertainties in the optical potential may allow us to hope for the correction of this feature.

The next step is to see if the target emission can explain pion production to the 2.plh staces. Let us first look at some characieristics of the target production mechanism. The effect of each of the terms in Eq. 12 is shown individually in Fig. 3.

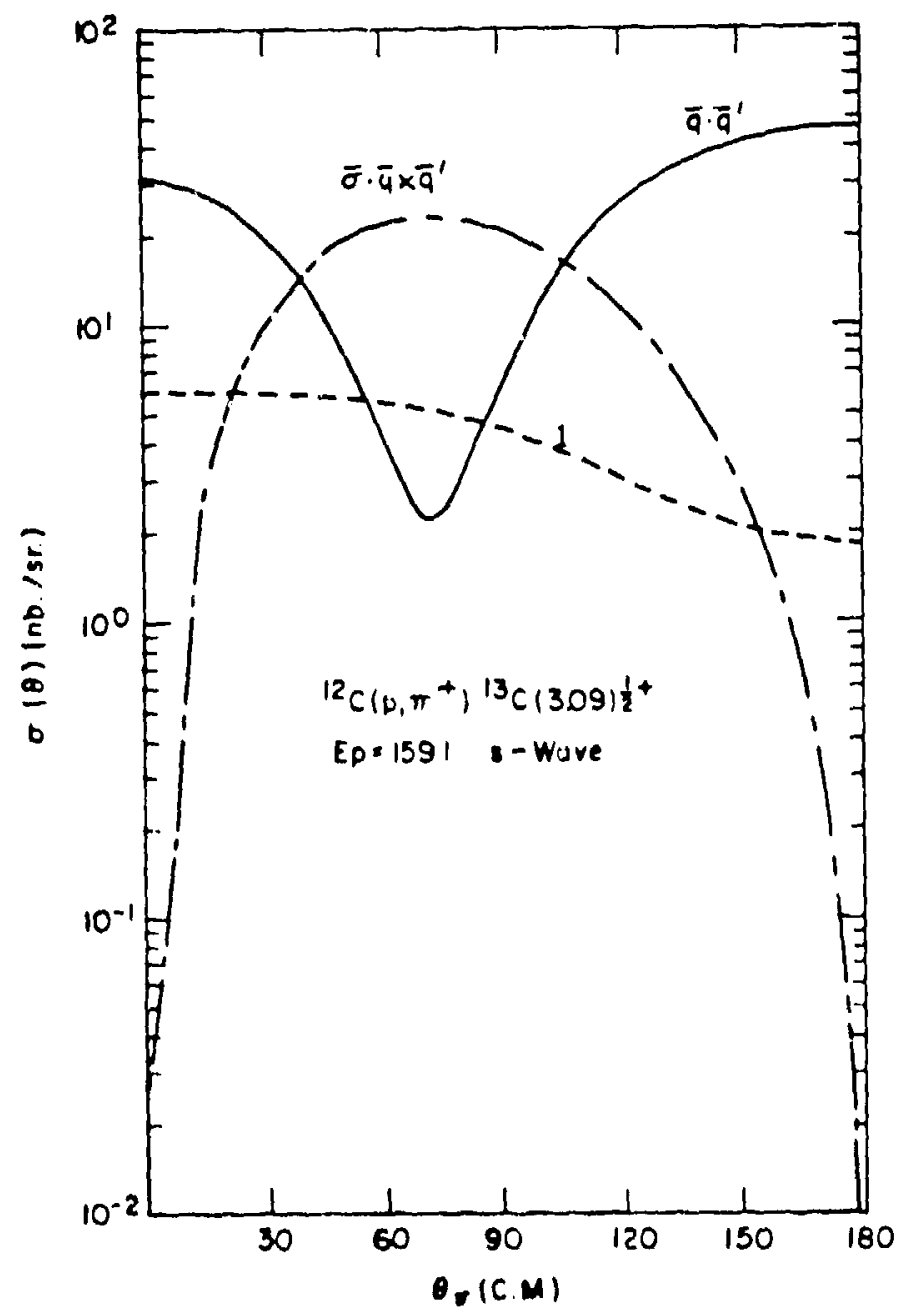

Fig. 3. Characteriftic whapes of a target emission calculation shnwi.s the dependence no the various parts of the $\pi$-nucleon $t$-matrix. 
The curve labled "l" contains only the S-wave pion-nucleon scattering and thus shows the form factor and distortion effects only. The other two curves show the $\cos ^{2} \theta$ and $\sin ^{2} \theta$ dependences as well. These features are very clear in the case presented, but not quite so clear in others. The spin dependent term is too large for the ${ }^{13} \mathrm{C}$ states. It is not known whether this is due to the fact that the pion-nucleon t-matrix should be modified in the nuclear medium to reduce the spin dependent piece, whether some feature of the calculation should be modified to reduce this term or if we are simply barking up the wrong graph. In any case, for the remainder of the comparisons with ${ }^{13} \mathrm{C} I$ shall set this term to zero.

The calculations are classified to some extent by the core excited state. For p-wave capture in the final state the positive parity states will be described by $0^{-}$or $2^{-}$(or higher in some cases) core states.

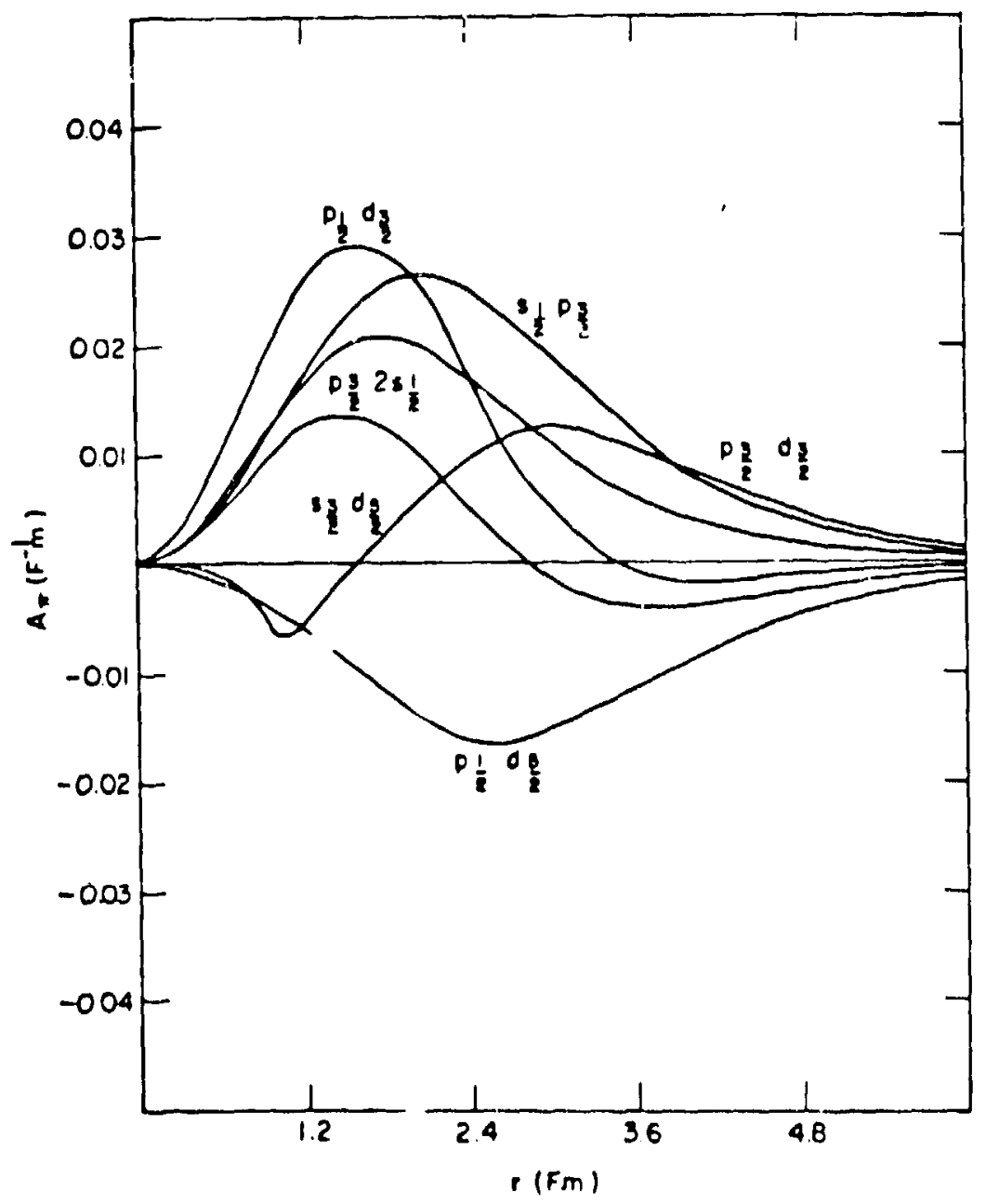

Fig. 4. The hole particle components entering into the structure of the 2 core state. 
The hole-partjcle pairs allighed for $0^{-}$are

${ }_{1 \mathrm{~s}}^{-1} \mathrm{l}_{2} \frac{1}{2}, \mathrm{p}_{\frac{1}{2}} 2 \mathrm{~s}_{\frac{1}{2}}$ sod $1 \mathrm{p}_{\frac{3}{2}} 1 \mathrm{~d}_{\frac{3}{2}}$ For 2 the available states

are $1 s_{\frac{1}{2}}^{-1} 1 p_{\frac{3}{2}}, 1 p_{\frac{3}{2}}^{-1} s \frac{1}{2}, 1 p_{\frac{1}{2}}^{-1} 1 d_{\frac{3}{2}}, 1 p_{\frac{1}{2}}^{-1} 1 d_{\frac{5}{2}}, 1 p_{\frac{3}{2}}^{-1} 1 d_{\frac{3}{2}}, 1 p_{\frac{3}{2}}^{-1} 1 d_{\frac{5}{2}}$

The hole-particle A functions for the $2^{-}$case are shown in figure 4.

The actual $A(r)$ used in Eq. 11 is : linear combination of these functions with coefficients given by the shell model. It is common for one or two of these basis functions to dominate. The shape of $A(r)$ has a large effect on modification of the angular distribution.

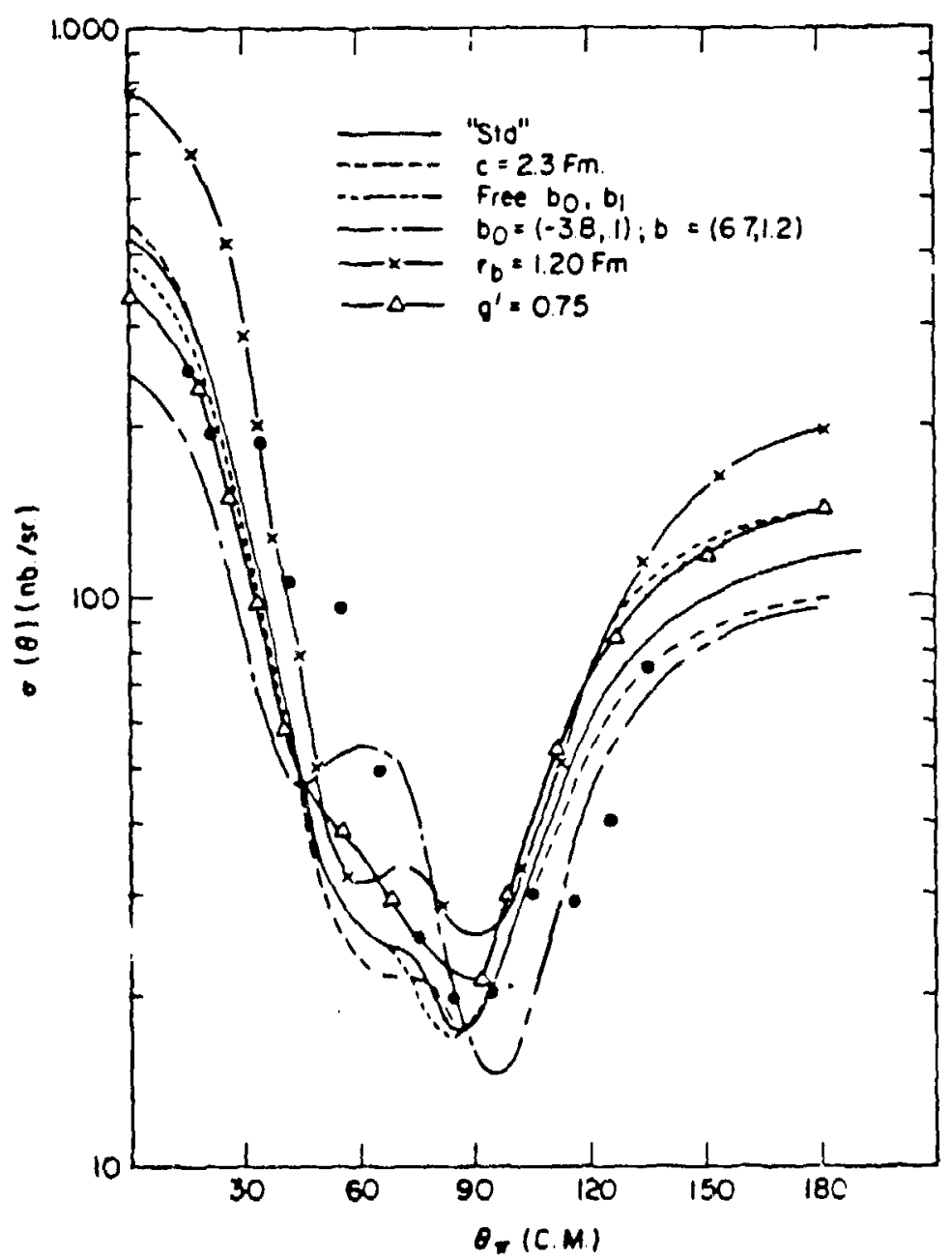

Fig. 5. The effect of variation of some of the input parameters on the angular distribution. The solid ("std") curve is calculated for c (the W-S radius for ${ }^{12} \mathrm{C}$ in the optical potential) $=2.41 \mathrm{fm}, \mathrm{b}=(2.7, .9)$ $b=(7.8, .5), r_{b}$ (radius of the $W-S$ well for computing the final bound s(ate) $=1.31 \mathrm{fm}$, and no enhancement :rom th: RPA corrections. 
The first state considered is the $8.86 \frac{5+}{2}$ state in ${ }^{13} \mathrm{C}$. This 1 state is dominated by components

$$
\begin{aligned}
& \left\{\left[\begin{array}{cc}
1 p^{-1} & 2 s \\
\frac{3}{2} & \frac{1}{2}
\end{array}\right]_{2} \times \frac{1 p}{2}\right\}_{\frac{5}{2}} \quad\left\{\left[\begin{array}{cc}
1 p^{-1} 1 d \\
\frac{3}{2} & \frac{5}{2}
\end{array}\right]_{2} \times \frac{1 p}{2}\right\} \frac{5}{2} \\
& \left\{\left[\begin{array}{cc}
1 \mathrm{p}^{-1} & 1 \mathrm{~d} \mathrm{f}_{\frac{5}{2}} \\
\frac{1}{2}
\end{array}\right]_{2} \times 1 \mathrm{p}_{\frac{3}{2}}\right\}_{\frac{5}{2}} \text {. }
\end{aligned}
$$

The 1pl/2 capture dominates and I show only these results in Fig. 5, i.e. all six components are included in the 2 core but only the pl/2 coupling is calculated. Figure 5 shows some angular distributions compared with data at $185 \mathrm{MeV}$. As can be seen the qualitative agreement is quite good with only moderate variations in shape corresponding to rather substantial modifications in the model.

Tigure 6 shows the angular distribution as a function of energy. Detailed agreement leaves a great deal to be desired but the dependence of magnitude on energy is good.

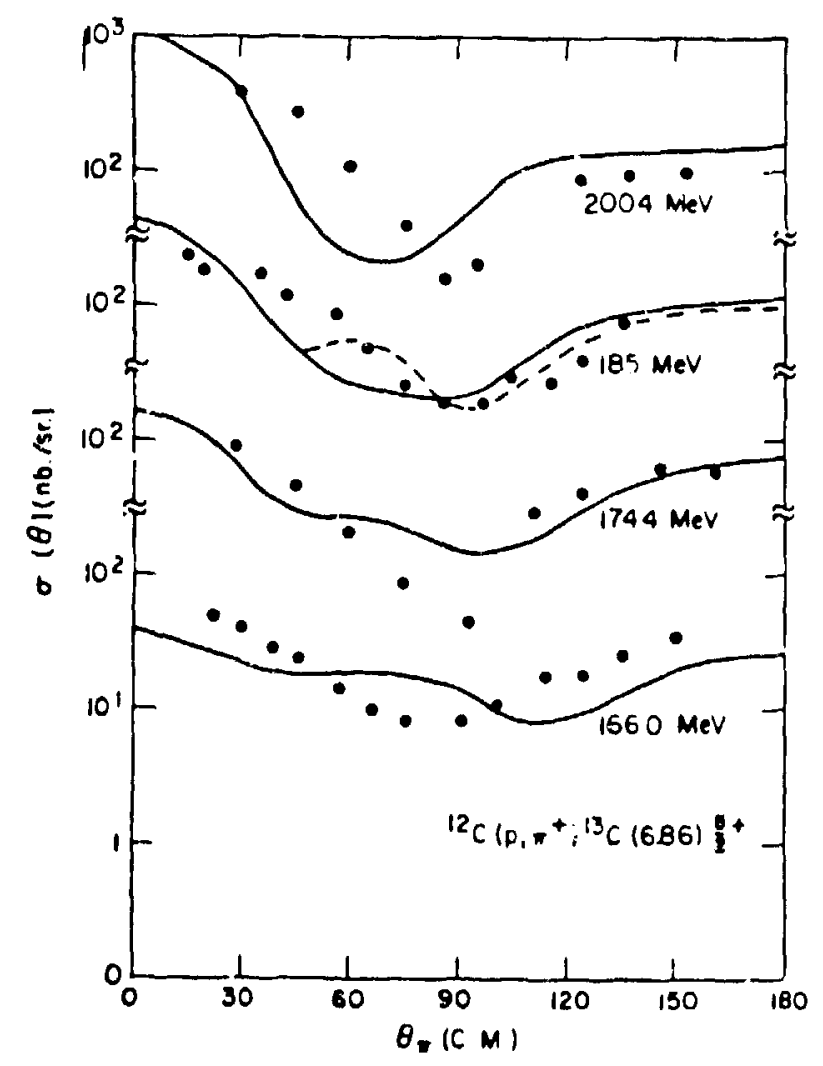

Fig. 6. Energy dependence of the angular distributions for the $6.86 \mathrm{MeV}$ state corresponding to the "std" curve in Figure 5. The dash-dot curve is the ame as in Figure 5 . 


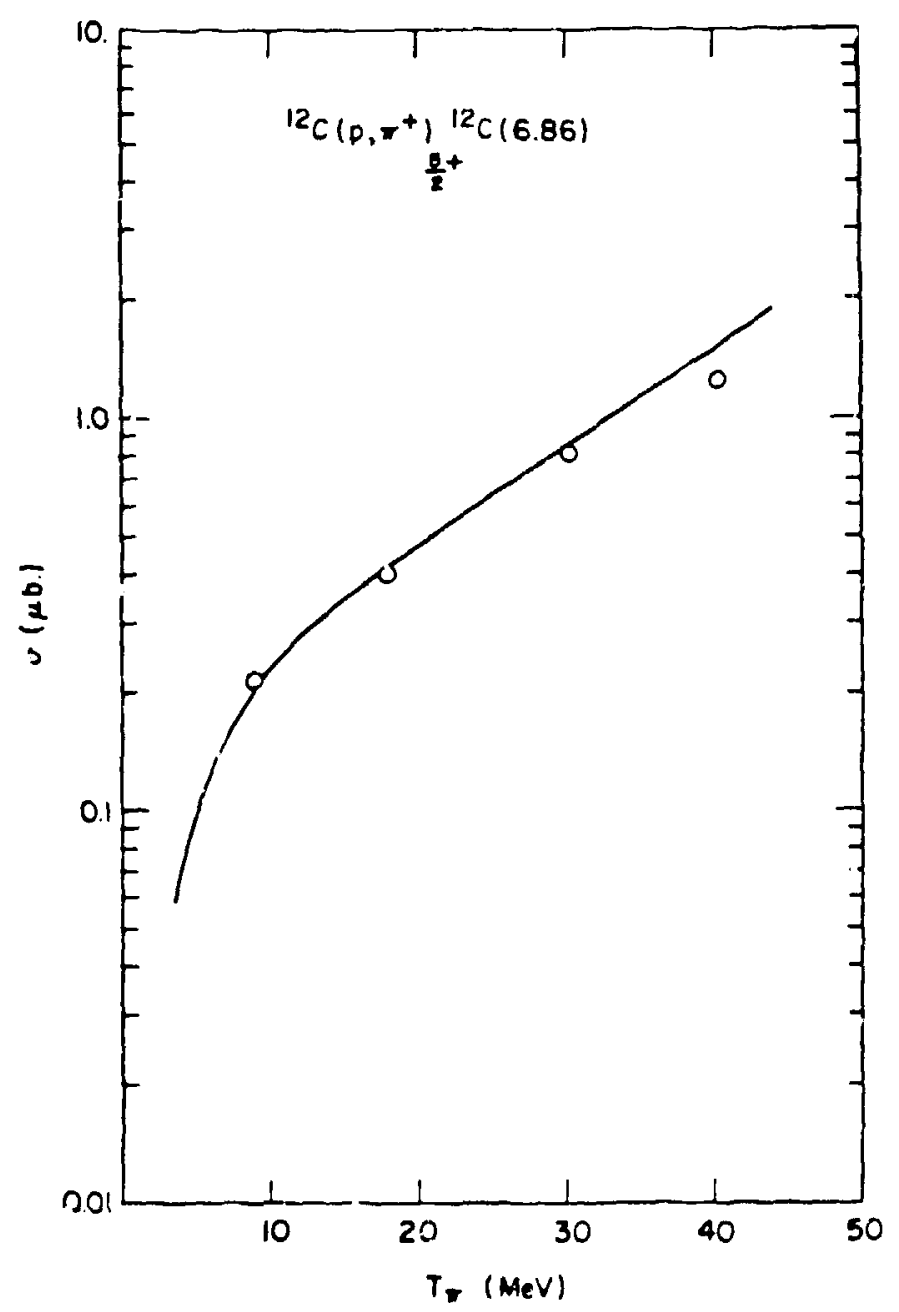

Figure 7. The energy dependence of the integrated cross section for the $6.85 \mathrm{MeV}$ state.

This is reflected in Fig. 7 where the integrated cross section is plotted. Thus we see that the gradual energy dependence is correctly given by this calculatjon.

The third step in the study of the separation hypothesis requires that the target emission calculation fail to give the energy dependence of a single particle state. For this case I bave chosen the $3.091 / 2^{+}$ final state in ${ }^{13} \mathrm{C}$. This state iz well sepresented by ${ }^{12} \mathrm{C} \times 25 \frac{1}{2}$. The target emission must destroy the core, however, so to sfore the state the captured particle must fill the bole. Thus since $\frac{1}{2}$ can be obtained by $0^{-} \times 1 p \frac{1}{2}$ or $2^{-} \times 1 \mathrm{p} \frac{3}{2}$ the dominant components are

$$
\left\{\left[\begin{array}{cc}
1 \mathrm{p}^{-1} & 2 \mathrm{~s} \\
\frac{1}{2} & \frac{1}{2}
\end{array}\right]_{0} \times 1 \mathrm{p}_{\frac{1}{2}}\right\}_{\frac{1}{2}}
$$


$\left\{\left[\begin{array}{cc}1 \mathrm{p}^{-1} & 2 \mathrm{~s} \\ \frac{3}{2} & \frac{1}{2}\end{array}\right]_{2} \times \mathrm{rp}_{\frac{3}{2}}\right\}_{\frac{1}{2}}$

Figure 8 show's the result of the calculation from $s$ - and $d-$ core states (all hole-particle states included;.

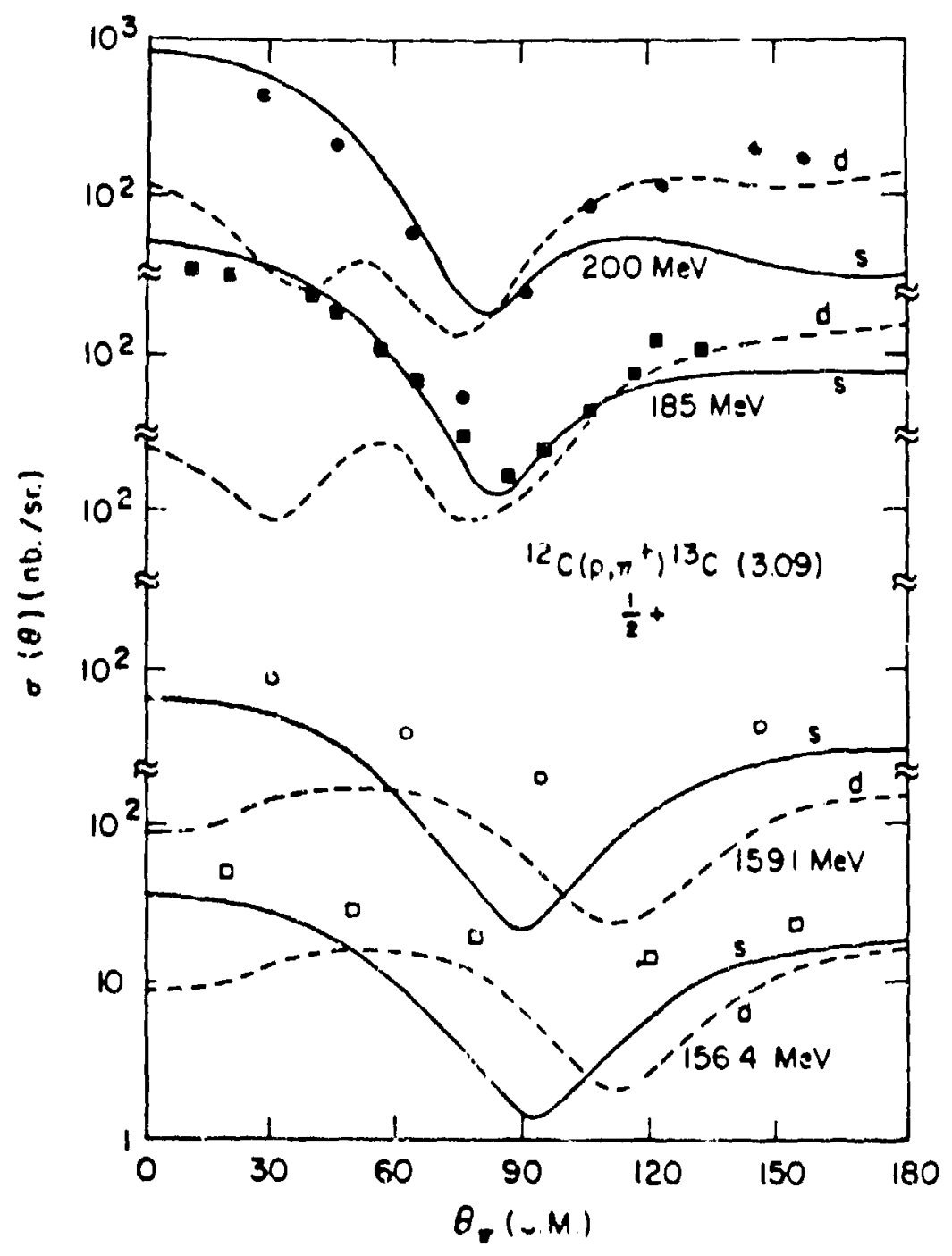

Figure B. Separate angular distributions for the 3,09 Mel' state.

Note that at high energy where the experimentel minumum is deep the minima of the two pieces rearly cosncide. At low enerey where the experamental aligular distribtion becomes much flater then the two theoretical minima de nut agier. It looks almest accidental that the i wave comes up in the lidte angles but recall that the relative strellgths will be given hy angulat mionentulu coefficients obtained by the decompesition of the single fartale state. 
In figure 9 the cross sections from the coherent sum of the s- and d-state core are shown. The general agreement with the angular distributions, in particular the energy dipendence of the forkard hemisphere cross sections, is largely satisfactory. In fact the agreement seems rather better for this single paricle state than for the $2 p 1 h$ itate.

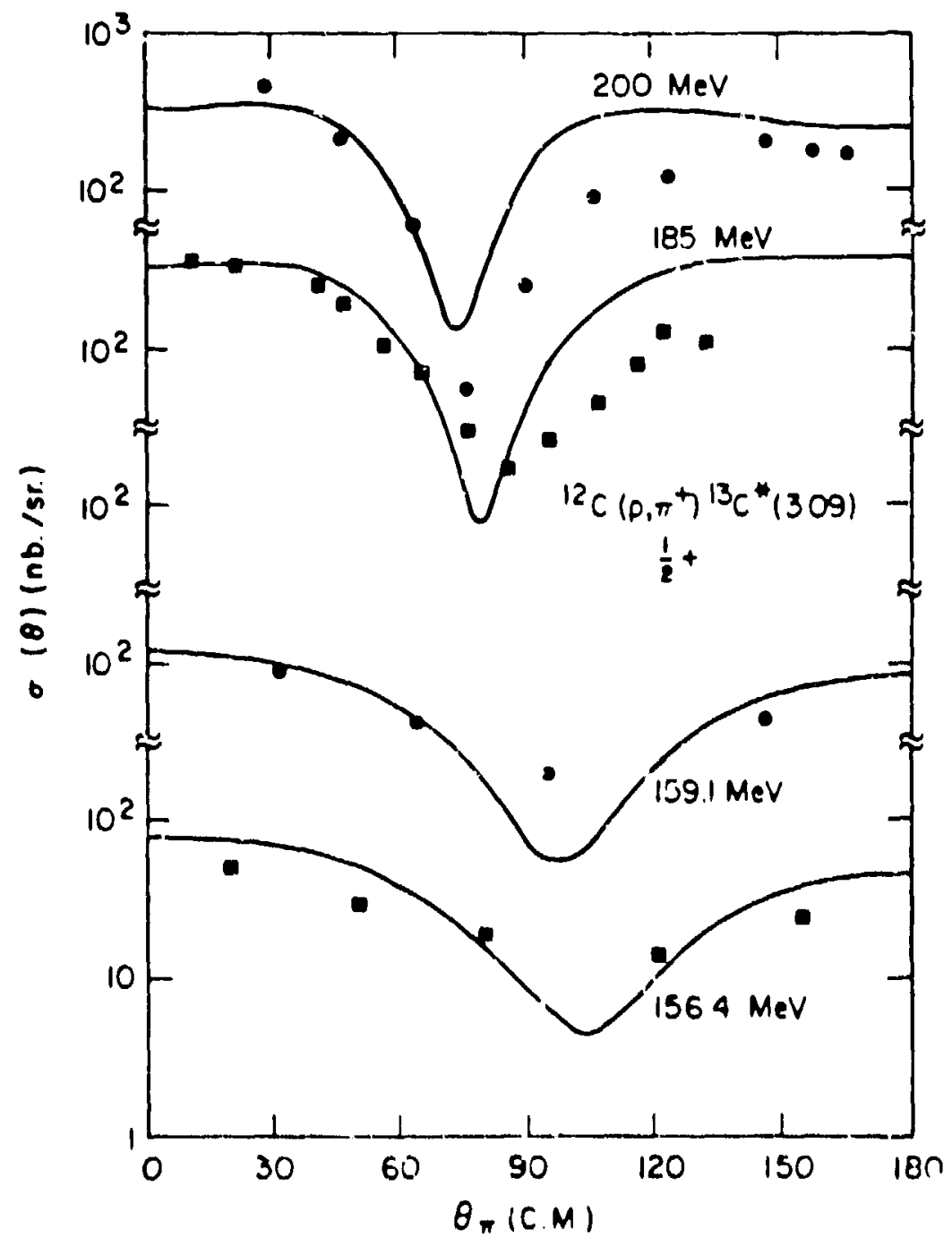

Figure 9. Combined angular distiblutons for the J.og Mel' state. 
The corresponding integrated cross sections are shown in figure :0.

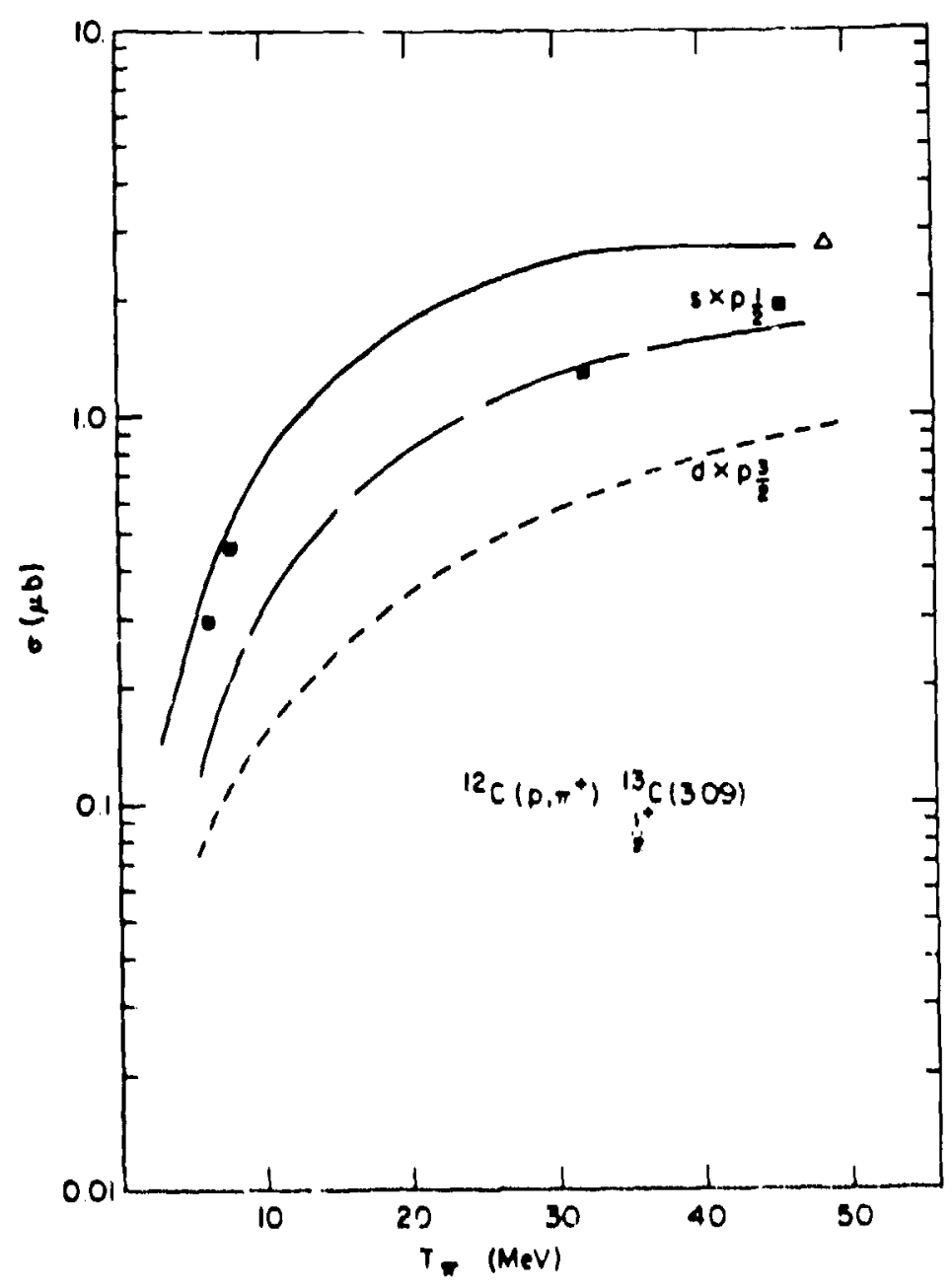

Figure 10. Separate and combined integreted cross sections for the 3.09 MoV slato.

We note that the s- and d- state energy dependenses are diflerent with the - sate sheibitz a depesidence like the single particle

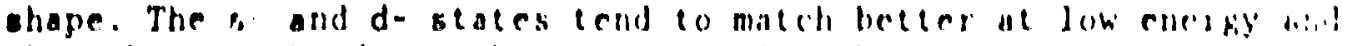
the coherence heids ug the cross nection there.

Thas we ner that. a separation of diagrams hy the energy urependence is not possible. This does not meno that a separation by strusture cannot le made, fust that the association of a dominalle of carget emission with 2 pllh states is not trur. 

A fairly typical asymmetry is shown in fig. 11. This is in
reasonable qualitative agreement with recent data. i6

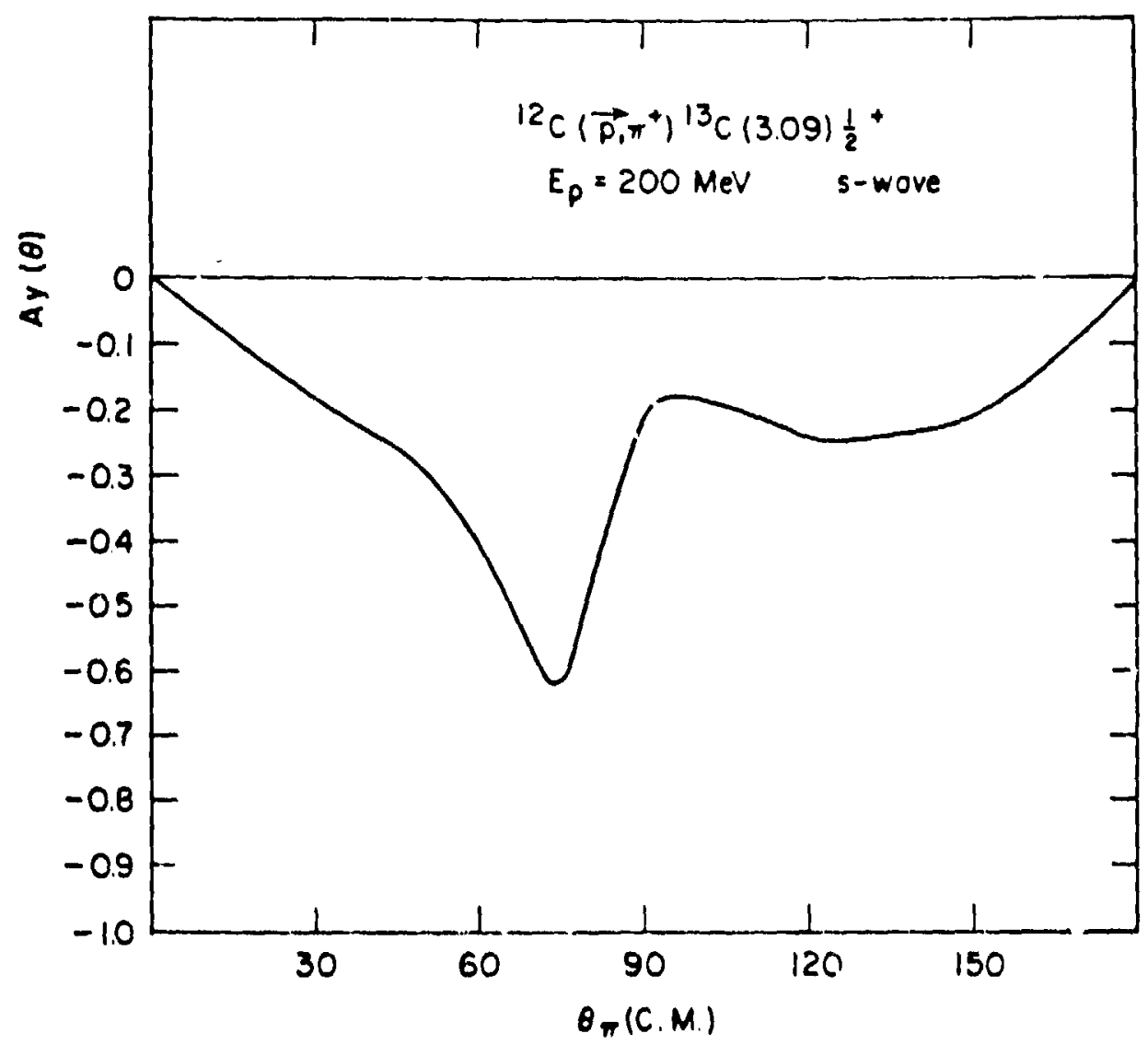

Figure 11. An example of asynmetry for the 3.09 Mel state. (mily a core is inleuded.

I shall briefly ansidgy one other sete, the $3.85 \mathrm{~s}$ alte ju ${ }^{13} \mathrm{C}$. Since this as a)so a state like the 6.86 one might expect the pion production to be similar. However, the parejele-hole composition is rather daterent. For this state the dominant feredicted) coneributors are

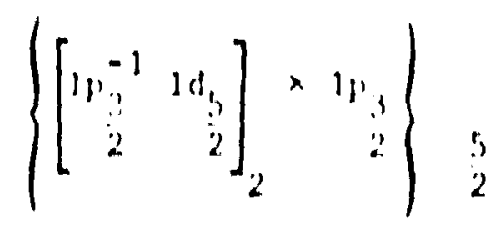




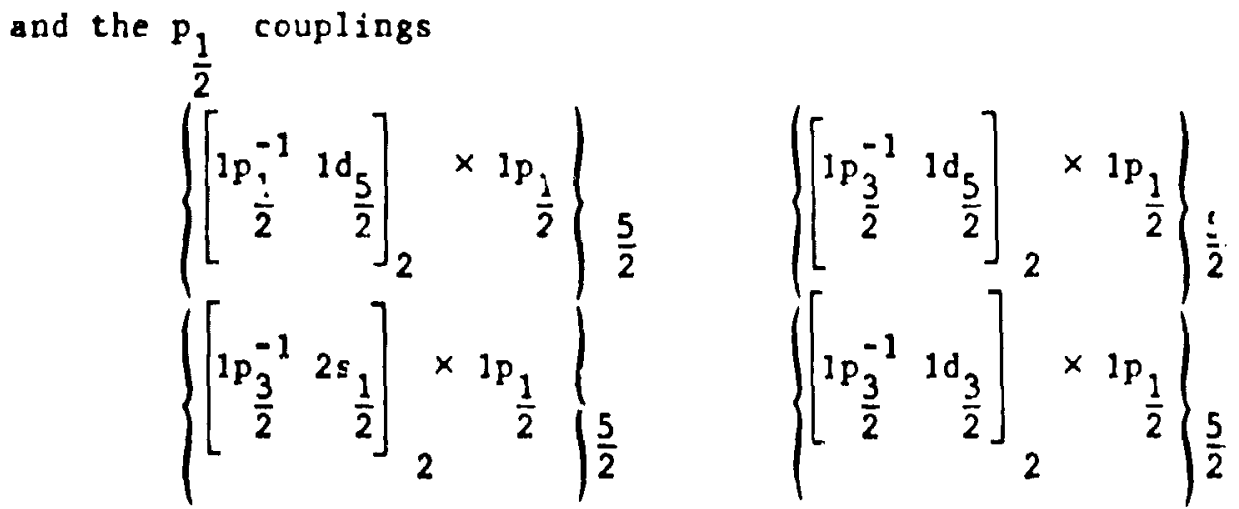

The characteristics of the measured angular distr:? utions are rather similar to the other $\frac{5}{2}$ state at 6.86 . Accoroing to the shell model the underlying structure is different. This difference is reflected in the angulis distributions of figure 12 . I unly give the separata pieces but it seems clear that, wile the general magnitude may not be too bad there is a great deal lacking in further agreenent. This leads us to think that a fair sensitivity to details of nuclear stucture exists.

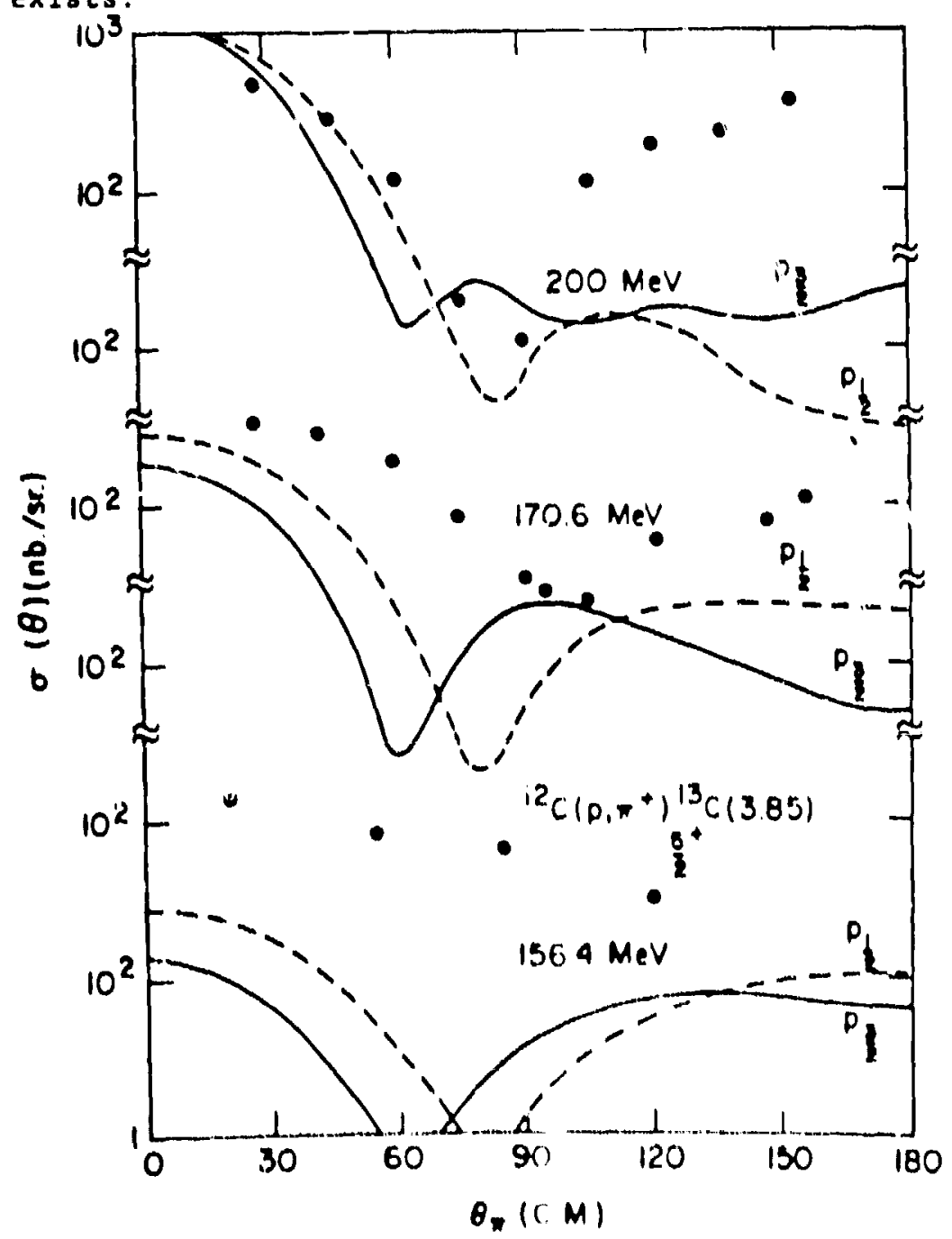

Figure. 12. Separate engular distributions for the 3.85 Mel etate. 
III. ENERGY DEPENDENCE IN ${ }^{209} \mathrm{Bi}\left(\mathrm{p}, \pi^{-}\right)^{210} \mathrm{At}$

Let me now consider a second study of the encrgy dependence of the target emission. A large part of negative pion production may be reasonably thought of as coming from target emission. The projectile emission may produce $\pi^{-}$by $\pi^{\circ}$ production followed by charge exchange and more exotic processes (such as $\Delta^{7+}$ mechanisms) can exist as well. I shall neglect these possibilities and assume that the negative pion is produced from one of the neutrons in the target sn that target emission is the total mechanism for negative pion production, admittedly an extreme point of view.

The reaction considered is ${ }^{209} \mathrm{Bi}\left(\mathrm{p}, \pi^{-}\right)^{210}$ At sollowed by the evaporation of neutrons. From the residual isotopic distribution, information about the original energy spectrum can be infered.

To make this connection some additonal assumptions will be needed. First we shall assume that the cross section to a state at a given energy can be represented (on average) by the cross section to a "typical" state.

For simplicity this state is taken to be a pure configuration.

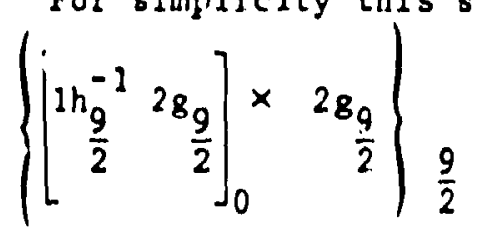

The cross sections calculated under this assumption are shown in figure 13. There are several interesting features in these curves.

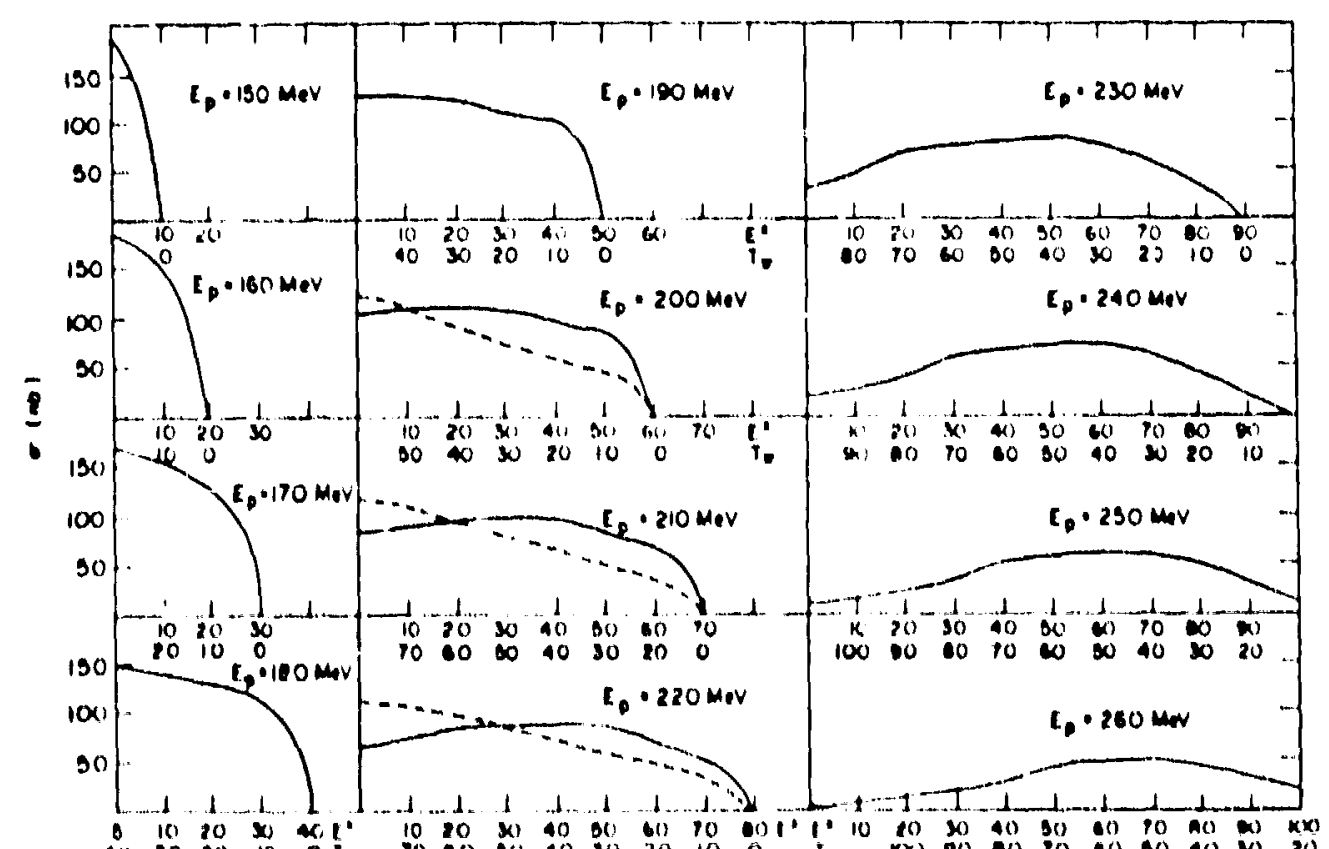

Higure 13. Calculated energy dependence to the "typucal" state in the $20 E_{B i}\left(p, n^{-}\right) 210 \mathrm{At}$ reaction. 
A rough idea of the isolopic abundance of $210 \mathrm{At}$ to bc left after this process can be estimated by integrating the first $10 \mathrm{MeV}$ of this function (it should be multiplied by a level density, and will be shortly). We note that this integral rises from threstold and then drops quickly, peaking at the surprisingly low energy of 10-20 MeV above threshold. This feature has already been noted both experimentally and theoretically. 17 Another feature of interest is the importance of the energy dependence of the pionnucleus optical potenial. The dotted curves show the energy variation of these basic cross sections fixing the optical parameters at their value at $50 \mathrm{MeV}$. The solid curves were calculated with all parameters kept fixed except for the imaginary part of b, which was taken as a linear combination of a constant plus the "free" value such that the elastic scattering is well represented at $50 \mathrm{MeV}$.

To calculate the spectral strength we must know the density of our "typical" states. In a simple inodel of equal spacing of single particle states, for bigb energies where Pauli exclusion cffects should be unimportant, the level density should vary as the energy raised to the number of "particles" minus one. Thus, for these 2plb sitites, the density should vary at $E^{2}$. To make the density a little more realistic at low energies the form

$$
\rho(E)=.004(E+15)^{2}
$$

(E in MeV) was used. The spectrum ralculated using this function is shown in figure 14.

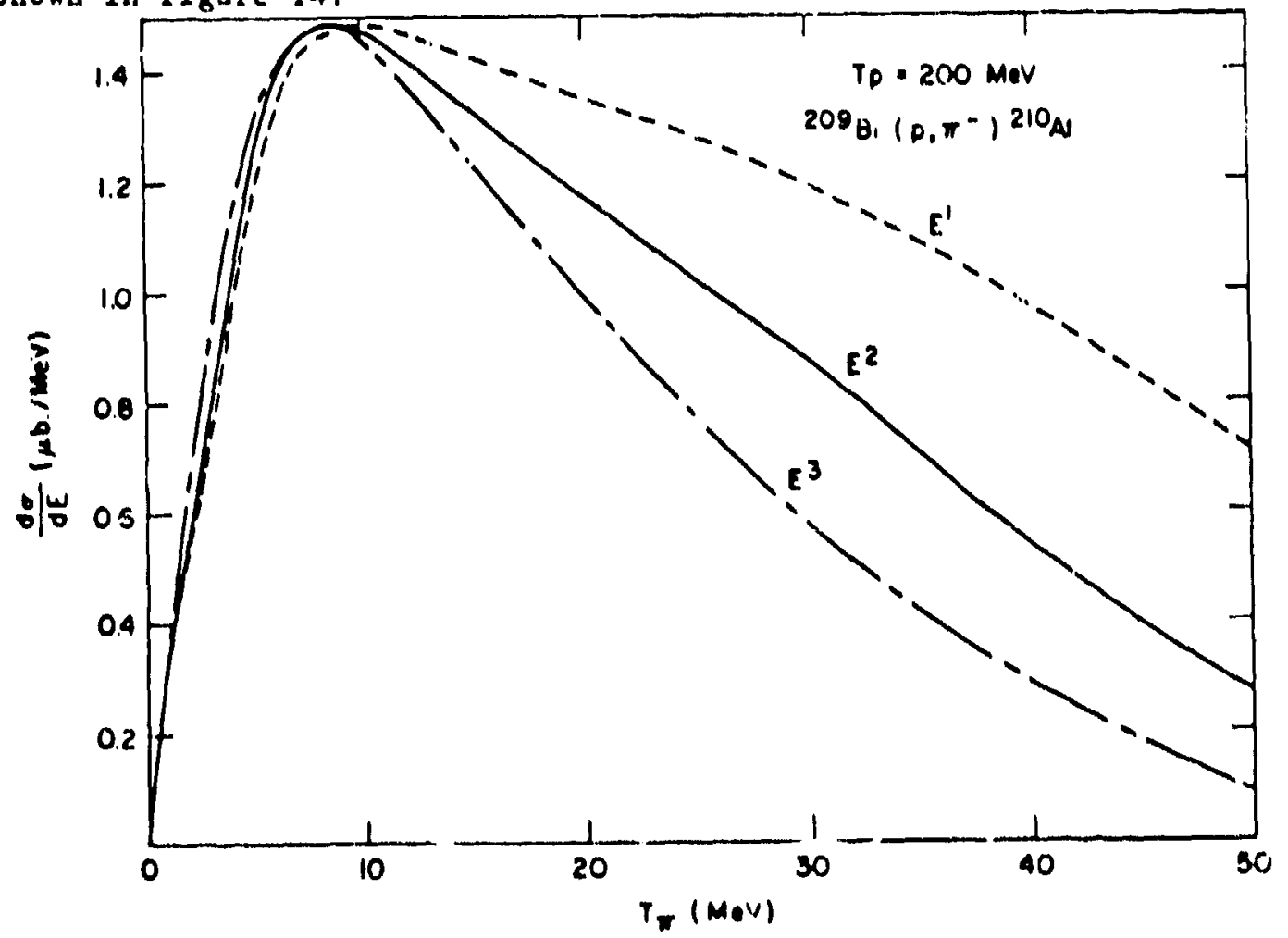

Figure 14. Calculated spectrum for negative pion production from protons on ${ }^{200} \mathrm{Bi}$. The curves labelid $E^{i}$ asd $E^{3}$ are chowil to entimate sensitivity to the level density ocumption. 
Also shown is the shape of the spectrum for two neighboring powers (normalized to the same peak value). Since the normalization of the level density and the spectroscopic factors are very crude the results can be considered as normalized to the total integrated data to all observed isotopes.

Following an evaporation of successive neutrons, the original $21{ }^{\circ} \mathrm{At}$ spectrum can be converted to a cross section for production of various isotopes. The comparison with data 18 is shown in figure 15 .

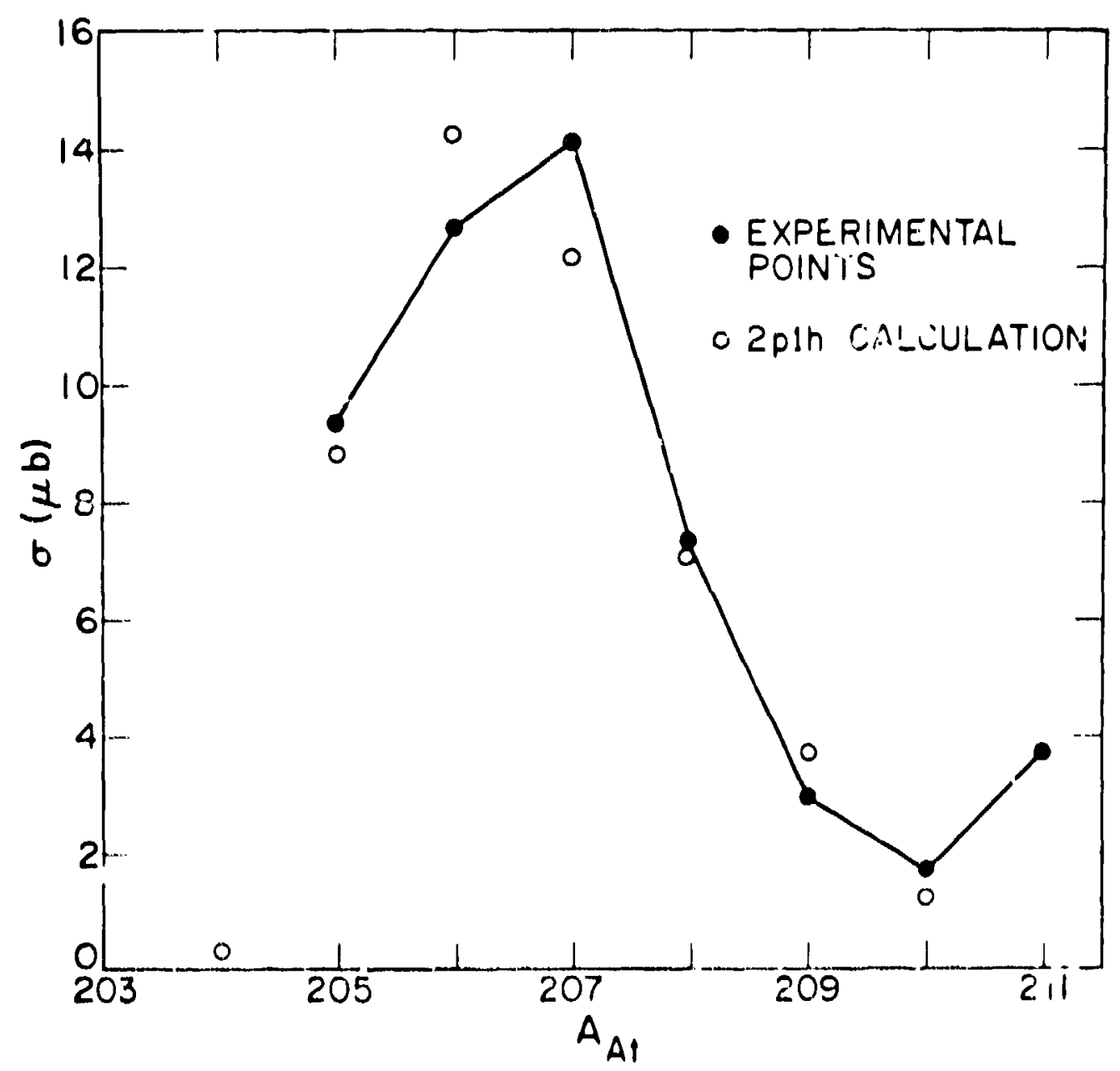

Figure 15. Comparison of the calculated relative isotope production cross aection (open circies) with the data of Ref. 18. 
The agreement is very good. The production of the various isotopes is extremely sensitive to the bombarding energy, especially near threshold. These cross sections are shown in figure 16 .

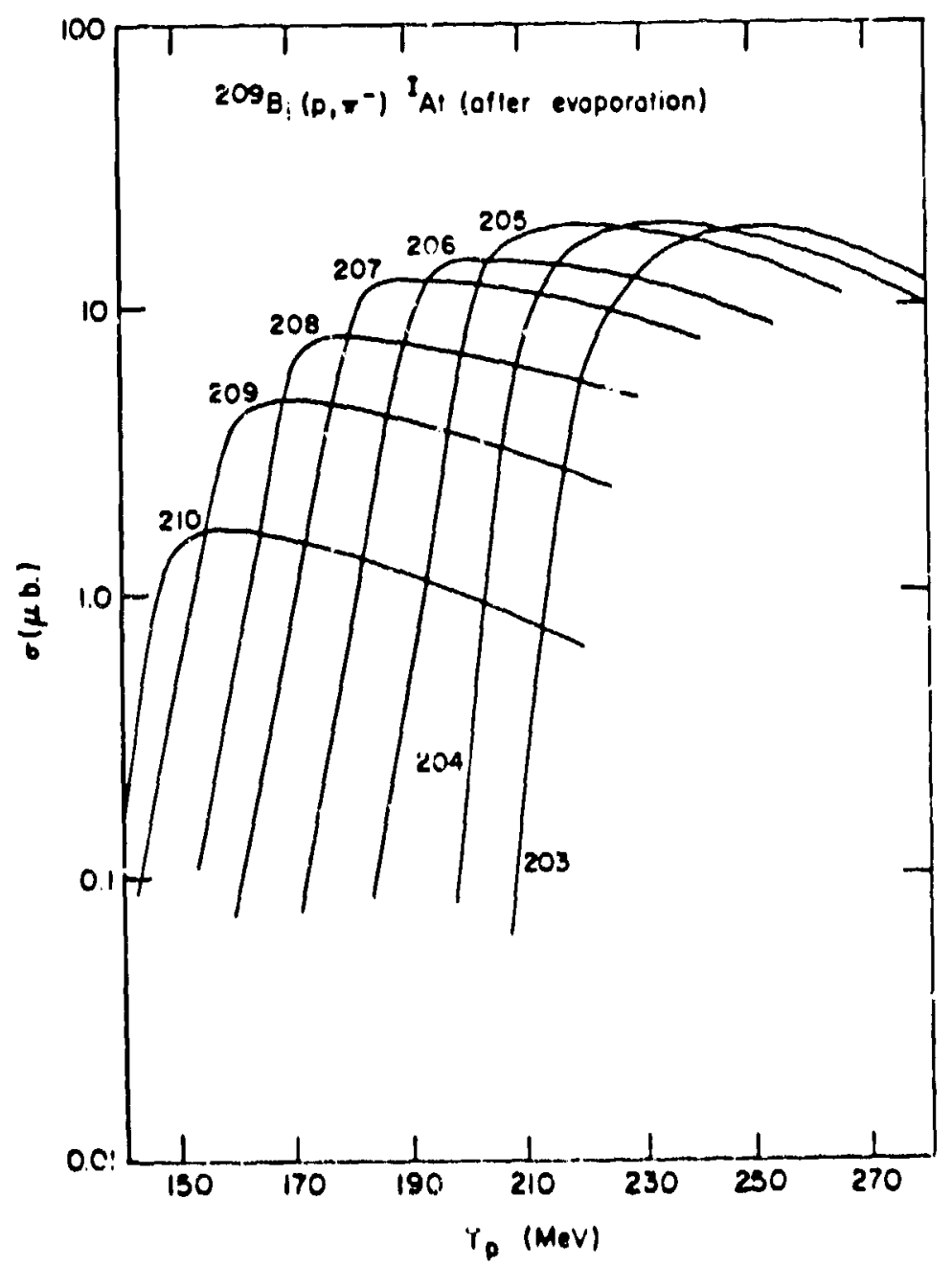

Figure 16. The dependence on proton hombarding encrgy of the cross section for production of At isotopes from a Bi target.

Clearly this technique provides all alternative to the use of Fermi motion for inclusive sul-threshold production of mesons. More detalled comparison with datn is necded to welcet between these two models. 
1) B. Goplen, W. R. Gibbs and E. L. Lomon, Phys. Rev. Lett. 32, 1012 (1974) B. M. Preedom et 31. Phys. Lett. 65B, 31 (1976).

2) D. O. Riska, M. Brack and W. Weise, Phys, Lett. 61B, 41 (1976).

3) J. Niskanan (these proceedings).

4) Bruce J. Verwest, Phys. Lett. 83B, 161 (1979)

5) M. Dillig (ihese proceeding ).

6) H. Toki and W. Heise, Z. Physik A295, 187 (1980), Phys. Rev. Lett. 42, 1034 (1979).

7) M. Ericson and J. Delozme, Phys. Lett. 76B, 182 (1978).

8) G. R. Satchler and R. M. Haybron, Phys. Lett. 11, 313, (1964).

9) J. R. Comfort and W. G. I.cye, Phys. Rey. Lett. $44,1656,(1980)$.

10) R. R. Whitehead. A. Watt, B. J. Cole and I. Morrison, Advances in Nuclear Physics Vol. 9, (Ed. by Baranger and Vogt) Page 123.

11) J. Dubach and k. C. Haxton, Phys. Rev. Lett. 41, 1453, (1978).

12) W. R. Gibbs, R. F. Gibson and G. J. Stephenson, Jr., Phys. Rev. Lett. 39, 1316 (1977). W. R. Gibbs, "Common Prohlens in Low-and Medium Energy Physi:s (Plenum Press New York and London 1979) p. 595.

13) H. Garcilazo and W. R. Gibbs, Niscl. Pbys. P.356, 248 (1981).

14) F. Sogo et al., Fhy =. Ty. C^4, 570 (1981).

15) M. P. Keating aus J. J. Wills, Phys. Rev. C7, 1336 (1973), M. Isangarides - Thesis - Univ. of Indiana (1978).

16) M. C. Green (trese proceedings)

17) T. E. Ward et al. Phys. Rev. C24, 588 (1981).

18) James L. Clark. Thesis - Carneigie-Mellon University, Sept. 1980. 